Int. J. Morphol., 36(1):201-205, 2018.

\title{
Somatotipo de Atletas de Taekwondo del Centro de Entrenamiento Regional de Valparaíso, Chile
}

\author{
Somatotype of Taekwondo Athletes at the Regional Training Center in Valparaíso, Chile
}

\author{
Gladys Orellana Lepe ${ }^{1}$; Alberto Warnier Medina ${ }^{1}$, Pedro Olivares Fernández ${ }^{1}$; \\ Rodrigo Yánez-Sepúlveda²; Tomás Herrera Valenzuela ${ }^{3,4}$ \& José Zapata Bastías ${ }^{1}$
}

ORELLANA, L. G.; WARNIER, M. A.; OLIVARES, F. P.; YÁNEZ-SEPÚLVEDA, R.; HERRERA, V. T. \& ZAPATA, B. J. Somatotipo de atletas de taekwondo del Centro de Entrenamiento Regional de Valparaíso, Chile. Int. J. Morphol., 36(1):201-205, 2018.

RESUMEN: El objetivo del estudio fue determinar el somatotipo de atletas de Taekwondo del Centro de Entrenamiento Regional (CER) de Valparaíso, Chile. Se evaluaron a los primeros cuatro clasificados según sexo (8 deportistas en total), hombres (18,75 \pm 2,62 años) y mujeres (18,50 \pm 1,91 años), competidores activos a nivel nacional e internacional en la disciplina deportiva. Para la evaluación se consideraron las normas establecidas por la International Society for the Advancement of Kinanthropometry (ISAK) y el grupo español de Cineantropometría (GREC). Los materiales utilizados fueron el kit Innovare básico CESCORF®, tallímetro y balanza TANITA®. Para determinar el somatotipo se consideró la propuesta de Heath \& Carter. Los resultados encontrados muestran en el género masculino una clasificación del somatotipo de mesomorfo balanceado (2,5-4,0-3,0), mientras que en el género femenino se clasificó como mesomorfo - endomorfo (3,3-3,6-2,3). Se concluye que en ambos géneros predomina el componente mesomorfia, las mujeres presentan un mayor nivel de endomorfia y un menor desarrollo de la mesomorfia en comparación con los hombres.

PALABRAS CLAVE: Somatotipo; Taekwondo; Alto rendimiento.

\section{INTRODUCCIÓN}

El Taekwondo es un deporte olímpico, dependiente de la Federación Mundial de Taekwondo (WT) creada en 1972, en el que sus deportistas hacen uso de las piernas debido a lo cual estas técnicas requieren un importante uso de la fuerza explosiva y agilidad (Valdes-Badilla et al., 2014; Pérez-Gutiérrez et al., 2015; Herrera-Valenzuela et al., 2016). Este deporte individual se caracteriza por ser acíclico, en el cual se compite durante 3 rondas de 2 minutos con un período de descanso de 1 minuto, se anotan puntos a través de golpes y patadas al torso y a la cabeza (Ball et al., 2011). En las últimas décadas, la evaluación de las características antropométricas y fisiológicas es implementada por entrenadores de esta disciplina deportiva (Arazi et al., 2016). En este sentido, la valoración de la composición corporal es un indicador indispensable para conocer la incidencia de las cargas, que constituye un aspecto a controlar en la preparación de los deportistas (Mesa Sánchez et al., 2015). Asímismo, Arazi et al. aclara que, independiente del tipo de deporte, tener habilidades físicas tales como características antropométricas y fisiológicas es un requisito previo para el éxito contra cualquier competición o torneo. En este sentido, es fundamental tener en cuenta que, para los entrenadores de esta disciplina es importante el planteamiento de objetivos a corto, mediano y largo plazo en sus programas de preparación con la suficiente argumentación y evidencia científica (Cardozo et al., 2017). En este sentido, es importante señalar que las descripciones y perfiles antropométricos son importantes de definir en el taekwondo y en otros deportes, tales como en el andinismo, basquetbol, canotaje, esgrima, gimnasia artística, judo, karate, tenis de mesa, tiro al arco, entre otros (Rodríguez et al., 2014), afirmando que el somatotipo es un indicador del alto rendimiento deportivo (Dantas \& Fernandes, 2005), siendo además, un excelente indicador de la forma y estructura del cuerpo humano (Vera et al., 2014), el cual cobra importancia, ya que, cada especialidad deportiva presenta una serie de exigencias que obliga, en la mayoría de los casos, a poseer una determinada anatomía en los deportistas con el fin de lograr un des-

\footnotetext{
${ }^{1}$ Escuela de Educación, Carrera de Pedagogía en Educación Física, Universidad Viña del Mar, Viña del Mar, Chile.

${ }^{2}$ Facultad de Filosofía y Educación, Pontificia Universidad Católica de Valparaíso, Valparaíso, Chile.

${ }^{3}$ Escuela de Ciencias de la Actividad Física, el Deporte y la Salud, Facultad de Ciencias Médicas, Universidad de Santiago de Chile, Santiago, Chile.

4 Escuela de Ciencias del Deporte, Facultad de Salud, Universidad Santo Tomás, UST, Chile.
} 
empeño deportivo óptimo (Rodríguez et al.). El somatotipo, es un método que describe una forma y composición corporal actual, expresada en la cuantificación de tres componentes (endomorfia, que expresa el contenido de grasa corporal, la mesomorfia el desarrollo del músculo esquelético y la ectomorfia la delgadez del cuerpo) relativo a la altura (Carter \& Heath, 1990; Ochoa Martínez et al., 2014). De cada uno de los tres componentes mencionados se obtiene la media y la desviación estándar, información que luego se ubica en una distribución gráfica bidimensional conocida como somatocarta (Aragón \& Fernández, 1995).

Para el cálculo del somatotipo el método más utilizado es el de Heath \& Carter (Carter \& Heath), el cual permite un estudio preciso del tipo físico ideal de cada deporte, siendo una excelente herramienta para utilizarse en el descubrimiento de talentos, además de permitir un continuo acompañamiento de los componentes somatotípicos durante una temporada competitiva. Este posee un $\mathrm{r}=0,98$, convirtiéndose así en un método seguro de evaluación (Toledo Fonseca et al., 2010). En base a lo mencionado, conocer los valores antropométricos del deportista de alto rendimiento en el taekwondo resulta relevante, ya que, existe una escasez de este tipo de investigaciones en Chile (Rodríguez et al.).

De acuerdo a lo señalado, el objetivo de este estudio es identificar el somatotipo de los atletas de taekwondo del Centro de Entrenamiento Regional (CER) de la región de Valparaíso en Chile.

\section{MATERIAL Y MÉTODO}

Muestra. El presente estudio contó con una muestra de ocho atletas de Taekwondo seleccionados del CER de la Región de Valparaíso, quienes participaron de manera voluntaria en este estudio previa firma y autorización en base a consentimiento informado. Todos los deportistas que constituyeron la muestra compiten en el deporte de alto rendimiento y están clasificados en los primeros lugares en la región, participaron cuatro hombres $(18,75 \pm 2,62$ años) y cuatro mujeres (18,50 $\pm 1,91$ años). Los criterios de inclusión de este estudio fueron: tener una trayectoria deportiva destacada dentro de campeonatos regionales, nacionales e internacionales en competencias de Taekwondo (WT); pertenecer y entrenar regularmente en el CER de la región de Valparaíso de Chile.

Materiales. Las evaluaciones se realizaron en el CER de la Región de Valparaíso en Chile, se utilizó un kit Innovare básico ${ }^{\circledR}$ CESCORF (Porto Bello, Brasil), el cual está compuesto por un plicómetro (presión+- $10 \mathrm{~g} / \mathrm{mm}^{2}$ ), un antropómetro $(164 \mathrm{~mm})$ y una cinta antropométrica (longitud $2 \mathrm{~m}$ ).
Adicionalmente, se utilizó un tallímetro portátil TANITA ${ }^{\circledR}$ (dimensiones: 300 x 250 x $2200 \mathrm{~mm}$ ) y una balanza TANITA ${ }^{\circledR}$ TBF 300 (Tokio, Japón) con capacidad máxima de 200 kg.

Protocolo. Previo consentimiento informado se evaluaron las medidas antropométricas acorde a los lineamientos de la International Society for the Avancement in Kineanthropometric (ISAK) y el grupo español de Cineantropometría (GREC). Dentro de los parámetros del somatotipo existen variables que permiten categorizar a un individuo de acuerdo a la masa muscular, masa grasa y los componentes óseos, las que generan tendencia a los siguientes componentes primarios: endomorfia, mesomorfia y ectomorfia.

Las mediciones necesarias para el cálculo del somatotipo de acuerdo al protocolo Heath \& Carter (Carter, 2002) son: estatura (cm), peso corporal $(\mathrm{kg})$, cuatro pliegues cutáneos (mm): tricipital, subescapular, supraespinal y pantorrilla, dos diámetros óseos $(\mathrm{cm})$; biepicondilar del húmero y biepicondilar del fémur y dos perímetros $(\mathrm{cm})$ : brazo flexionado en tensión y pantorrilla. Se consideraron las recomendaciones de la Declaración de Helsinki para la investigación en seres humanos (World Medical Asociation, 2013).

Análisis estadístico. Para determinar el somatotipo se utilizó estadística descriptiva, se consideraron los estadísticos media y desviación stándar. Los datos fueron divididos según género (mujeres $n=4$, hombres $n=4$ ). Para el análisis de datos se utilizaron los softwares Microsoft ${ }^{\circledR}$ Office Excel 2013 (Redmond, Washington, USA), GraphPad Prism ${ }^{\circledR}$ versión 6.0 (La Joya California, USA) y el Software Somatotype $^{\circledR}$ (San Diego, CA, EE.UU).

\section{RESULTADOS}

Se consideraron los elementos esenciales para el cálculo del somatotipo. A continuación se expresan los resultados de las variables básicas y antropométricas obtenidos en la muestra (Figs. 1 y 2; Tablas I y II).

La Tabla I muestra los valores promedio y desviación estándard de las características básicas tanto de las damas como de los varones evaluados.

En la Tabla II se presentan los resultados de mujeres y hombres. Se visualizan pliegues cutáneos, diámetros óseos y perímetros considerados para la clasificación del somatotipo, se aprecian los resultados obtenidos en los componentes y la clasificación obtenida en el somatotipo. 


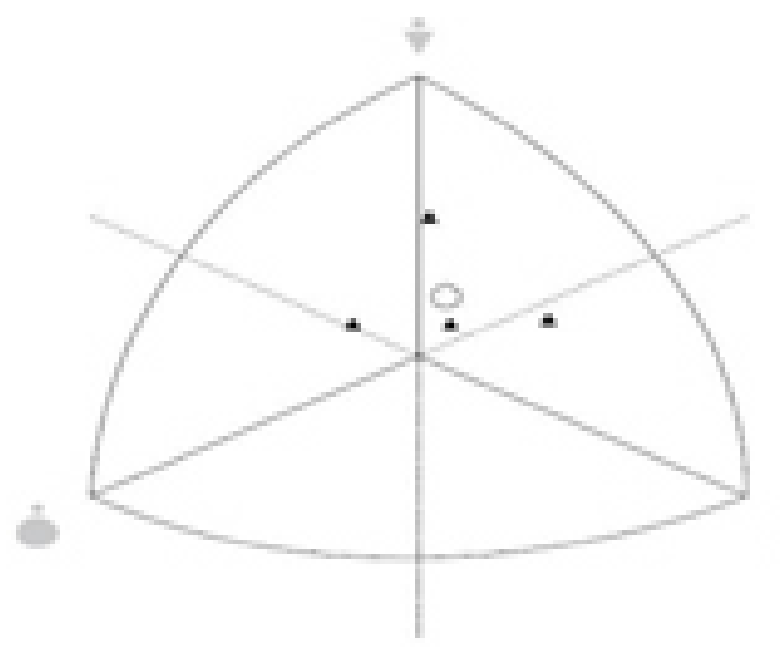

Fig. 1. La somatocarta del grupo de los varones presenta un somatotipo promedio con clasificación mesomorfo balanceado $(2,5-4,0-3,0)$.

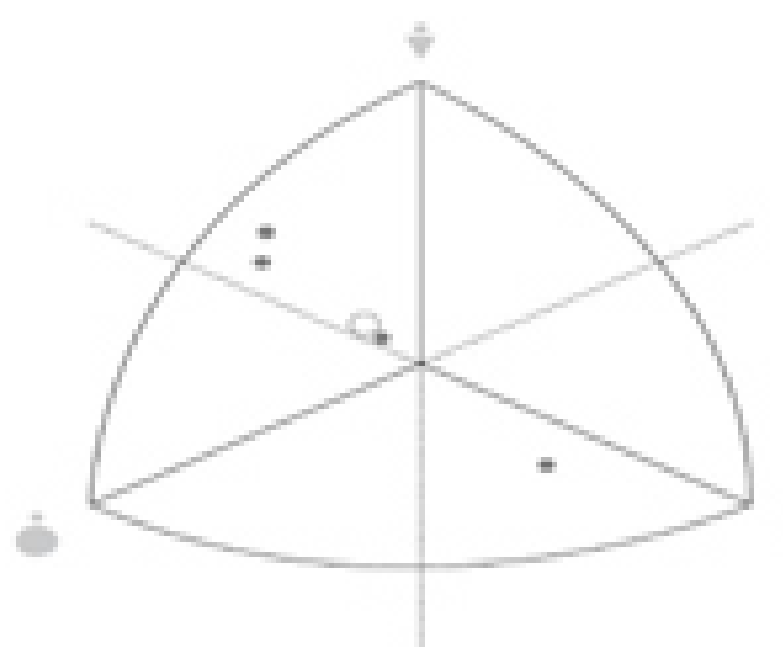

Fig. 2. La somatocarta del grupo de las mujeres expresa una clasificación mesomorfo - endomorfo (3,3-3,6-2,3).

Tabla I. Características básicas de la muestra.

\begin{tabular}{lrr}
\hline Variables & \multicolumn{1}{c}{ Mujeres } & \multicolumn{1}{c}{ Hombres } \\
\hline Edad (años) & $18,50 \pm 1,91$ & $18,75 \pm 2,62$ \\
Peso (kg) & $61,48 \pm 6,40$ & $68,00 \pm 8,22$ \\
Talla (mt) & $1,66 \pm 0,05$ & $1,76 \pm 0,05$ \\
IMC (Peso/Talla $\left.{ }^{2}\right)$ & $22,38 \pm 2,80$ & $22,03 \pm 1,80$ \\
\hline
\end{tabular}

Tabla II. Variables para el cálculo del somatotipo evaluadas.

\begin{tabular}{llcr}
\hline & Variables & Mujeres & Hombres \\
\hline \multirow{4}{*}{ Pliegues (mm) } & Tricipital & $13,50 \pm 3,42$ & $8,03 \pm 2,53$ \\
& Subescapular & $9,50 \pm 1,91$ & $9,95 \pm 2,78$ \\
& Supraespinal & $9,23 \pm 2,05$ & $8,70 \pm 5,94$ \\
& Pantorrilla & $7,38 \pm 2,43$ & $5,15 \pm 1,24$ \\
Perímetros $(\mathrm{cm})$ (ros $(\mathrm{cm})$ & $5,80 \pm 0,56$ & $6,45 \pm 0,34$ \\
& Humeral & $8,75 \pm 0,24$ & $9,73 \pm 0,79$ \\
& Femoral & $26,90 \pm 2,79$ & $30,13 \pm 1,11$ \\
& Brazo flexionado en tensión & $37,23 \pm 1,75$ & $35,83 \pm 1,53$ \\
& Pantorrilla & $3,35 \pm 1,84$ & $2,50 \pm 1,07$ \\
& Endo & $3,65 \pm 0,86$ & $4,05 \pm 0,69$ \\
& Meso & $2,33 \pm 1,60$ & $3,00 \pm 0,79$ \\
& Ecto & Mesomorfo endomorfo & Mesomorfo Balanceado \\
\hline
\end{tabular}

\section{DISCUSIÓN}

En los resultados obtenidos de esta investigación, se evidencia que el grupo de atletas de taekwondo masculino del CER posee un somatotipo mesomorfo balanceado (2,5-4,13,0 ), valores distintos a los obtenidos en las atletas de taekwondo femenino, quienes tienen un somatotipo mesomorfo - endomorfo (3,4-3,7-2,3). Un estudio realizado por Panchi Sánchez (2010) quien evaluó a 27 varones taekwondistas mexicanos, evidenció un somatotipo endo mesomorfo $(4,2-5,1-2,0)$, similares a los encontrados en 46 atletas de taekwondo varones evaluados donde el somatotipo es endo - mesomorfo (3,5-4,6-2,8) (Shariat et al., 2017), en los cuales los valores de endomorfia y mesomorfia son mayo- 
res que los resultados encontrados en el presente estudio, mientras que la ectomorfia es menor en comparación a los resultados obtenidos en seleccionados de taekwondo chilenos quienes también presentan una diferencia en su clasificación del somatotipo en relación a atletas de taekwondo cubanos que poseen un somatotipo meso - ectomórfico (Betancourt León et al., 2009). Otro estudio realizado en hombres españoles de diversas categorías, se obtuvo que el somatotipo es diferente en algunas categorías de peso, en $-58 \mathrm{~kg}$ el somatotipo fue ecto - mesomorfo (1,7-3,9$3,6)$, en $-68 \mathrm{~kg}$ fue ecto - mesomorfo (1,8-4,3-3,3), en -80 $\mathrm{kg}$ fue ecto - mesomorfo $(2,2-4,5-2,9)$ y en $+80 \mathrm{~kg}$ fue endo - mesomorfo (3,0-5,5-1,9) (Pons et al., 2015). Solo la categoría $-80 \mathrm{~kg}$ se acerca a los valores obtenidos en los atletas de taekwondo evaluados en este estudio. El somatotipo de la muestra masculina chilena fue mesomorfo balanceado, clasificación diferente según los valores obtenidos en el estudio realizado con atletas de taekwondo españoles donde el somatotipo fue ecto - mesomorfo (2,25,4-2,8) (Falcó et al., 2008), en atletas argentinos fue ecto - mesomorfo (2,0-4,7-2,9) (Lentini et al., 2004) y en mexicanos fue ecto - mesomorfo (1,4-4,1-3,2) (Martínez-Sanz et al., 2011). Por otro lado, Bridge et al. (2014) señala que el somatotipo de atletas seleccionados de taekwondo coreanos fue ecto - mesomorfo (1,4-4,1-3,2), el de atletas de taekwondo estadounidenses de nivel internacional el somatotipo fue mesomorfo - ectomorfo $(1,6-4,5-4,6)$, el de alemanes fue ecto - mesomorfo (3,0-4,7-3,8), el de taekwondistas cubanos fue ecto - mesomorfo $(1,8-4,4-3,5)$ $\mathrm{y}$ el de atletas de taekwondo turcos es ectomorfo balanceado (2,6-2,6-3,5). De acuerdo a estos resultados, los valores de ectomorfia en los atletas chilenos de taekwondo son menores que los taekwondistas mexicanos, coreanos, estadounidenses, alemanes, cubanos y turcos, siendo mayor en comparación a los atletas españoles. A nivel formativo se han encontrado clasificación mesomorfo balanceado (2,7-5,0-2,3) (Godoy-Cumillaf et al., 2015) al igual que los atletas evaluados en esta investigación, sin embargo, en niveles formativos existen valores más elevados en endomorfia y mesomorfia y menores en ectomorfia en relación a la muestra masculina de este estudio, lo cual, podría ser consecuencia del desarrollo deportivo propio del atleta de alto rendimiento en el taekwondo.

En cuanto a la muestra femenina evaluada en esta investigación, el somatotipo es similar al de las seleccionadas argentinas de la misma disciplina deportiva (Lentini et al.), quienes poseen un somatotipo mesomorfo endomorfo (3,3-3,7-2,5), presentando disminución en el valor de endomorfia, aumento en la ectomorfia, mientras que en mesomorfia se mantiene igual. Resultados parecidos se encontraron en taekwondistas españolas (3,6-3,82,2) (Pons et al.). Por otro lado, Bridge et al. señala que el somatotipo de atletas de taekwondo turcas de nivel internacional fue mesomorfo - balanceado $(3,1-3,4-3,1)$, el de estadounidenses fue meso - ectomorfo $(2,1-3,2-4,0)$ y el de alemanas correspondió a mesomorfo balanceado (4,0$4,2-3,5)$, clasificaciones que resultan distintas a las obtenidas en los resultados de la muestra femenina de este estudio.Las taekwondistas del centro de alto rendimiento (CAR) de Chile presentaron una clasificación endo mesomorfo (3,1-4,2-2,3) (Rodríguez et al.), quienes solo presentan valores mayores en mesomorfia en relación a las deportistas de este estudio.

\section{CONCLUSIÓN}

Los resultados del estudio permiten establecer el somatotipo de los atletas de taekwondo del Centro de Entrenamiento Regional (CER) región de Valparaíso, Chile, a partir de esto se concluye que tanto en el sexo femenino como en el masculino presentan en promedio una predominancia del componente mesomorfia, las mujeres presentan mayor desarrollo de la endomorfia y menor desarrollo de la mesomorfia que los hombres, lo que se traduce en una diferencia en la clasificación del somatotipo en ambos grupos.

Todos los resultados antes expuestos contribuyen al conocimiento científico en el área del entrenamiento deportivo, y más específicamente en la disciplina del Taekwondo, al conocer valores reales de los atletas que actualmente participan en diversas competencias en categoría adulto en el alto rendimiento, y también para generar un parámetro de valores estándares que pueda servir como guía en las referencias antropométricas de los deportistas de este nivel en esta disciplina deportiva.

ORELLANA, L. G.; WARNIER, M. A.; OLIVARES, F. P.; YÁNEZ-SEPÚLVEDA, R.; HERRERA, V. T. \& ZAPATA, B. J. Somatotype of taekwondo athletes at the Regional Training Center in Valparaíso, Chile. Int. J. Morphol., 36(1):201-205, 2018.

SUMMARY: The aim of the study was to determine the somatotype of taekwondo athletes at the Regional Training Center (CER) in Valparaíso, Chile. The first four were classified according to sex ( 8 athletes in total), men $(18.75 \pm 2.62$ years) and women (18.50 \pm 1.91 years), all of them active competitors in the discipline nationally and internationally. For the evaluation, the standards established by the International Society for the Advancement of Kinanthropometry (ISAK) and the Spanish Group of Cineanthropometry (GREC) were considered. The 
materials used were CESCORF® basic Innovare kit, TANITA® weight/height scale. To determine the somatotype, the Heath \& Carter proposal was considered. The results showed that in men a classification of the somatotype of balanced mesomorph (2.5-4.0$3.0)$, while in women it was classified as mesomorphic endomorphic (3,3-3,6 - 2,3). It is concluded that in both sexes the mesomorphic component predominates, women present a higher level of endomorphy and a lower development of mesomorphy compared to men.

formance.

KEY WORDS: Somatotype; Taekwondo; High per-

\section{REFERENCIAS BIBLIOGRÁFICAS}

Aragón, L. \& Fernández A. Fisiología del Ejercicio. San José de Costa Rica, Universidad de Costa Rica, 1995.

Arazi, H.; Hosseinzadeh, Z. \& Izadi, M. Relationship between anthropometric, physiological and physical characteristics with success of female taekwondo athletes. Turk. J. Sport Exerc., 18(2):69-75, 2016.

Ball, N.; Nolan, E. \& Wheeler, K. Anthropometrical, physiological, and tracked power profiles of elite taekwondo athletes 9 weeks before the Olympic competition phase. J. Strength Cond. Res., 25(10):2752-63, 2011.

Betancourt León, H.; Aréchiga Viramontes, J. \& Carvajal Veitía, W. Estimación antropológica de la forma corporal de atletas elites cubanos de deportes olímpicos de combate. Antropol, 19:23-32, 2009.

Bridge, C. A.; Ferreira da Silva Santos, J.; Chaabène, H.; Pieter, W. \& Franchini, E. Physical and physiological profiles of taekwondo athletes. Sports Med., 44(6):713-33, 2014.

Cardozo, L. A.; Vera-Rivera, D. A.; Conde-Cabezas, O. A. \& Yañez, C. A. Aspectos fisiológicos de deportistas elite de taekwondo: Una revisión narrativa. Rev. Esp. Educ. Fis. Deportes, (418):35-46, 2017.

Carter, J. \& Heath, B. Somatotyping: Development and Application. New York, Cambridge University Press, 1990.

Carter, J. E. The Heath-Carter Somatotype Method. San Diego, San Diego State University Syllabus Service, 2002.

Dantas, E. \& Fernandes, J. Atividade Física em Ciencias da Saúde. Rio de Janeiro, Shape, 2005.

Falcó, C.; Estevan, I.; Álvarez, O. \& Martos, J. Análisis Descriptivo del Perfil Antropométrico del Taekwondista de Alto Nivel: Un Estudio sobre el Equipo Nacional Español Masculino. Valencia, III Simposio Internacional de Ciencias de la Actividad Física. y el Deporte, 2008.

Godoy-Cumillaf, A. E. R.; Valdes-Badilla, P. A.; Soler, N. S.; CarmonaLópez, M. I. \& Fernández, J. J. Anthropometric characteristics of adolescents from different sports training schools. Int. J. Morphol., 33(3):1065-70, 2015

Herrera-Valenzuela, T.; Valdés-Badilla, P.; Franchini, E.; Ferreira da Silva Santos, J.; Ramírez-Campillo, R.; García-Hermoso, A.; Durán-Aguero, S. \& Castaneda-Gómez, J. Effects of multi-component training on the physical fitness of young taekwondo athletes. Ido Mov. Cult. J. Martial Arts Anthropol., 16(4):31-7, 2016.

Lentini, N. A.; Gris, G. M.; Cardey, M. L.; Aquilino, G. \& Dolce, P. A. Estudio somatotípico en deportistas de alto rendimiento de Argentina. Arch. Med. Deporte, 21(104):497-509, 2004.

Mesa Sánchez, L.; García Hernández, T. R.; Linares, F. \& Aguilera Ramírez, B. Caracterización de la composición corporal de las atletas de taekwondo del estado Cojedes en el período de preparación general. Lect. Educ. Fis. Deportes, 20(211), 2015.

Ochoa Martínez, P. Y.; Hall López, J. A.; Alarcón Meza, E. I.; Arráyales
Millán, E. M. \& Sánchez León, R. Somatotype profile and body composition of players from the Mexican professional basketball league. Int. J. Morphol., 32(3):1032-5, 2014.

Panchi Sánchez, C. Tendencia de somatotipo en competidores de taekwondo de la selección del Distrito Federal. Análisis retrospectivo. Efdeportes.com/Revista Digital, 15(144), 2010.

Pons, V.; Riera, J.; Galilea, P. A.; Drobnic, F.; Banquells, M. \& Ruiz, O. Características antropométricas, composición corporal y somatotipo por deportes. Datos de referencia del CAR de San Cugat, 1989-2013. Apunts Med. Esport, 50(186):65-72, 2015.

Rodríguez, P. X.; Castillo, V. O.; Tejo, C. J. \& Rozowski, N. J. Somatotipo de los deportistas de alto rendimiento de Santiago, Chile. Rev. Chil. Nutr., 41(1):29-39, 2014.

Shariat, A.; Shaw, B. S.; Kargarfard, M.; Shaw, I. \& Lam, E. T. C. Kinanthropometric attributes of elite male judo, karate and taekwondo athletes. Rev. Bras. Med. Esporte, 23(4):260-3, 2017.

Toledo Fonseca, C. L.; Roquetti Fernandes, P. \& Fernandes Filho, J. Analysis of anthropometrical profile of Brazilian junior volleyball team. Int. J. Morphol., 28(4):1035-41, 2010.

Vera, Y.; Chávez, C.; David, A.; Torres, W.; Rojas, J. \& Bermúdez, V. Características morfológicas y somatotipo en futbolistas no profesionales, según posición en el terreno de juego. Rev. Latinoam. Hipertens., 9(3):13-20, 2014.

World Medical Association (WMA). Declaration of Helsinki - Ethical Principles for Medical Research Involving Human Subjects. Fortaleza, World Medical Association (WMA), 2013.

\author{
Dirección para correspondencia \\ Rodrigo Yañez Sepúlveda \\ Facultad de Filosofía y Educación \\ Pontificia Universidad Católica de Valparaíso \\ El Bosque 1290 \\ Viña del Mar \\ CHILE
}

Email: fach.efi@gmail.com

Recibido : 30-11-2017

Aceptado: 07-12-2017 